\title{
Synchronization control of interacting oscillatory ensembles by mixed nonlinear delayed feedback
}

\author{
Oleksandr V. Popovych ${ }^{1}$ and Peter A. Tass ${ }^{1,2}$ \\ ${ }^{1}$ Institute of Neuroscience and Medicine-Neuromodulation (INM-7) and Virtual Institute of Neuromodulation, Research Center Jülich, \\ 52425 Jülich, Germany \\ ${ }^{2}$ Department of Stereotaxic and Functional Neurosurgery, University of Cologne, 50924 Cologne, Germany
}

(Received 26 April 2010; revised manuscript received 24 June 2010; published 9 August 2010)

\begin{abstract}
We propose a method for the control of synchronization in two oscillator populations interacting according to a drive-response coupling scheme. The response ensemble of oscillators, which gets synchronized because of a strong forcing by the intrinsically synchronized driving ensemble, is controlled by mixed nonlinear delayed feedback. The stimulation signal is constructed from the mixed macroscopic activities of both ensembles. We show that the suggested method can effectively decouple the interacting ensembles from each other, where the natural desynchronous dynamics can be recovered in a demand-controlled way either in the stimulated ensemble, or, intriguingly, in both stimulated and not stimulated populations. We discuss possible therapeutic applications in the context of the control of abnormal brain synchrony in loops of affected neuronal populations.
\end{abstract}

DOI: 10.1103/PhysRevE.82.026204

PACS number(s): 05.45.Xt, 87.19.L-

\section{INTRODUCTION}

Synchronization is known to play an important role in many fields of the natural sciences ranging from physics and biology to neuroscience and medicine [1-8]. For example, a well-coordinated firing of neurons within separate loops and pathways in the brain is one of the main mechanisms for neuronal signaling and information processing [3,9]. In contrast, an abnormal neuronal synchronization results in a pathological functioning of neuronal networks characteristic for many neurological disorders such as essential tremor or Parkinson disease (PD) [10-15]. An excessive synchronization has been found among neurons in basal ganglia (BG) in parkinsonian patients $[16,17]$ and animal models of PD $[18,19]$. In contrast, under physiological conditions the neuronal firing in the BG is almost always uncorrelated [18-20]. Well-pronounced synchronuos neural oscillations strongly correlate with the limb tremor, one of the clinical hallmarks of PD $[16,21,22]$. In fact, it has been shown that collective synchronized neuronal activity in the BG in the theta band $(3-7 \mathrm{~Hz})$ actually drives the limb tremor $[23,24]$. In addition, akinesia and rigidity, the other main symptoms of PD, strongly correlate with the amount of pathological synchronization in the beta band $(9-35 \mathrm{~Hz})$ [25].

Pathological output of the BG significantly disrupts the normal functioning of the entire basal ganglia-thalamocortical motor loop [10-14]. Many recent studies provide evidence that the interaction between the individual and the collective dynamics of neurons in the BG and in cortical areas is strengthened in the parkinsonian state [26-28]. Strong coherence has also been found between tremor muscle activity and motor cortex activity at single and double tremor frequencies [29,30], where the cortical activity led that of the tremor. Noteworthy, the cortical activity at these frequencies is driven by synchronized collective neuronal dynamics from the $\mathrm{BG}$, which, in turn, receives the feedback response from the cortex [27]. Such a driveresponse functional loop promotes the emergence of undesirable large-scale neuronal synchronization within the entire basal ganglia-thalamo-cortical circuit $[11,12,14,31]$.

In this paper, we present a method for the control of such drive-response loops of interacting synchronized populations of oscillators. We illustrate a potential application to deep brain stimulation (DBS), which is the standard therapy for medically refractory movements disorders, e.g., Parkinson disease $[13,32]$. High-frequency (HF) DBS has been developed empirically and appears to strongly modify the neuronal firing by blocking or overactivating neuronal action [33-35]. It is a reversible technique and has a much lower rate of side effects than lesioning with thermocoagulation [36]. However, the therapeutic effect of HF DBS can be limited or can decrease over time, and can be accompanied by side effects [37-39]. Hence, there is a significant clinical need for less invasive stimulation techniques, which restore physiological, desynchronized dynamics of oscillatory neurons and counteract large-scale pathological interactions between neural networks.

During the last decade several methods have been developed for the suppression of synchronization in oscillatory networks, which are based on phase resetting principles $[4,40,41]$ or feedback stimulation [42-47]. The former methods utilize short resetting stimulation pulses $[4,48]$ and require a repetitive administration keeping the stimulated population in a permanent transient between synchronized and desynchronized states-provided synaptic plasticity and, hence, antikindling mechanisms [49] are not taken into account. The time-delayed feedback techniques work at a minimal amount of stimulation force and effectively stabilize the desynchronized state, but require a clean measured signal representing the macroscopic dynamics of the stimulated oscillators.

The situation is much more difficult in the case of two oscillatory ensembles interacting according to the driveresponse coupling schema, where the response ensemble gets synchronized because of the permanent strong forcing originating from the drive population, as, e.g., in Parkinson disease. Moreover, it is also difficult to obtain a clean signal from a selected single source in the brain (e.g., with the standard recording techniques such as MEG or EEG, see 
Ref. [50]) in the presence of several active neuronal clusters, especially, if they are synchronized. The measured and reconstructed signal may contain a mixture of components coming from both populations participating in the synchronized drive-response loop of neuronal clusters.

In this paper, we consider such a stimulation setup and suggest a method where synchronization of two interacting networks of coupled oscillators can be controlled by stimulation with mixed nonlinear delayed feedback. We consider the case where only one of the interacting ensembles is stimulated with a signal constructed from the mixed mean fields of both ensembles. We show that depending on the level of mixing either one of the populations or, which is most intriguing, both of them can effectively be desynchronized and decoupled from each other. We illustrate the method with two examples of coupled limit cycle and phase oscillators. The latter model is used to reveal the important dynamical properties of the suggested control method and quantify the extent of phase synchronization among stimulated oscillators. To study the underlying stimulation mechanism, we also consider a simple system of two coupled limitcycle oscillators to model the dynamics of the mean fields of the stimulated ensembles. The main asymptotic properties of the decoupling and desynchronizing effect of the stimulation with the mixed nonlinear delayed feedback will be described. Finally, we illustrate the applicability of the method by considering a system of interacting ensembles of relaxation van der Pol oscillators.

\section{LIMIT-CYCLE OSCILLATORS: STIMULATION PROTOCOL}

We consider two populations of limit-cycle oscillators

$$
\begin{gathered}
\dot{z}_{j}=\left(a_{j}+i \omega_{j}-\left|z_{j}\right|^{2}\right) z_{j}+C_{1} Z+C_{21} W, \\
\dot{w}_{k}=\left(b_{k}+i \xi_{k}-\left|w_{k}\right|^{2}\right) w_{k}+C_{2} W+C_{12} Z+S,
\end{gathered}
$$

where $z_{j}(t)$ and $w_{k}(t)$ are the complex variables of the individual limit-cycle oscillators representing a topological normal form of a supercritical Andronov-Hopf bifurcation [51]. The amplitudes and the natural frequencies of the oscillators of the first (second) ensemble are given by the parameters $\sqrt{a_{j}}\left(\sqrt{b_{k}}\right)$ and $\omega_{j}\left(\xi_{k}\right)$, respectively. The oscillators are coupled within and between the ensembles via the mean fields $Z$ $=N_{1}^{-1} \sum_{j=1}^{N_{1}} z_{j}$ and $W=N_{2}^{-1} \sum_{k=1}^{N_{2}} w_{k}$, where $N_{1}$ and $N_{2}$ are the numbers of elements in the ensembles. We assume that the coefficients for the coupling within populations $\left(C_{1}, C_{2}\right)$ and between populations $\left(C_{12}, C_{21}\right)$ are chosen such that the following holds. The coupling within population 2 is weak $\left(C_{2}\right.$ is small), so that-isolated from population $1-$ no synchronization emerges in population 2 . In contrast, the coupling in population 1 is strong enough $\left(C_{1}\right.$ is large) to cause synchronization within population 1 and-mediated by the interpopulation coupling-also within population 2 as well as between both populations.

The second, driven ensemble of Eq. (1) is stimulated with signal $S(t)$ which is a nonlinear combination of instantaneous and delayed mean fields of both ensembles. To construct the

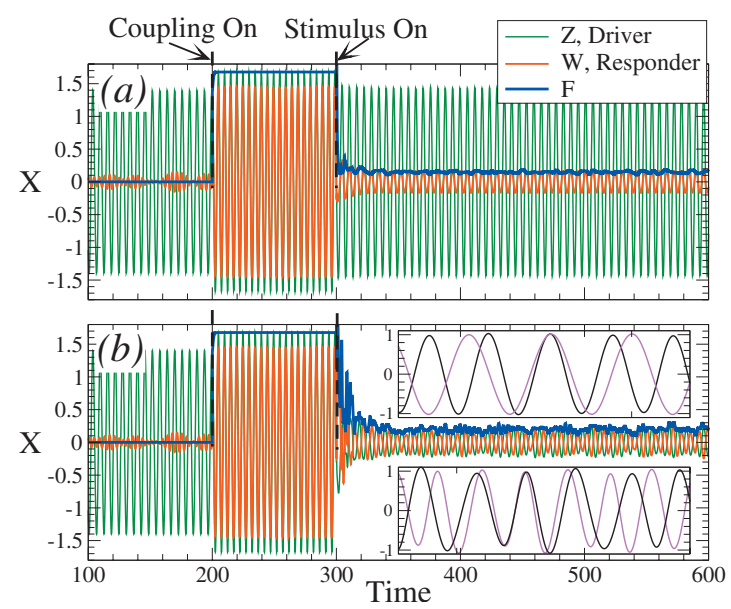

FIG. 1. (Color) Desynchronization and decoupling of interacting populations (1) by stimulation with the mixed NDF (2). The time courses of the real parts ( $X$-coordinates) of the complex mean fields $Z$ (green curves) and $W$ (red curves) and total driving force $F=\mid S$ $+C_{12} Z$ applied to the driven population (blue curves) are shown. In the course of time, the inter-population coupling with $C_{12}=C_{21}=1$ and the stimulation with $K=300$ are switched on for $t>200$ and $t$ $>300$, respectively. In the inserts in (b) trajectories of two selected oscillators from the first [upper subplot] and from the second [lower subplot] ensembles (1) are shown in the stimulated regime. Number of oscillators $N_{1}=N_{2}=100$. The frequencies $\omega_{j}$ and $\xi_{k}$ are Gaussian distributed around the mean frequencies $\Omega_{0}=2 \pi / T_{1} \approx 1.05\left(T_{1}=6\right)$ and $\Xi_{0}=2 \pi / T_{2} \approx 1.57\left(T_{2}=4\right)$, respectively, with the same deviation $\sigma=0.1$. The mixing (a) $\varepsilon=0$ and (b) $\varepsilon=0.73$, and the other parameters $a_{j}=b_{k}=1, C_{1}=1, C_{2}=0$, and $\tau=3.0$.

stimulation signal, we assume that a linear combination $S_{\varepsilon}$ of the macroscopic dynamics (mean fields) of both interacting population is taken. Then the measured signal is nonlinearly combined according to the rule of nonlinear delayed feedback (NDF) $[43,45]$

$$
\begin{gathered}
S(t)=K S_{\varepsilon}^{2}(t) S_{\varepsilon}^{*}(t-\tau), \\
S_{\varepsilon}(t)=\varepsilon Z(t)+(1-\varepsilon) W(t),
\end{gathered}
$$

where $K$ is the stimulus amplification coefficient, $\tau$ is the time delay, and the asterisk denotes complex conjugacy. The level of mixing of the mean fields $Z$ and $W$ within the stimulation signal is given by the parameter $\varepsilon$. If $\varepsilon=0$, only the driven and stimulated population 2 is measured, and if $\varepsilon=1$, only the drive population 1 contributes to the stimulation signal. For intermediate values of the mixing $\varepsilon \in(0,1)$ the mixed dynamics of both ensembles is used as stimulation signal. We study the impact of the level of mixing $\varepsilon$ on the macroscopic dynamics of ensembles (1) controlled by the mixed NDF (2).

Numerical simulations of ensembles (1) reveal several important effects of the stimulation with the mixed NDF (2) (Fig. 1). The onset of inter-population coupling at $t=200$ leads to large-amplitude oscillations of the mean fields of both, drive [Fig. 1, green curves] and response [Fig. 1, red curves] ensembles, characteristic for strong in-phase synchronization $[2,5]$. Then the onset of the stimulation at $t$ 

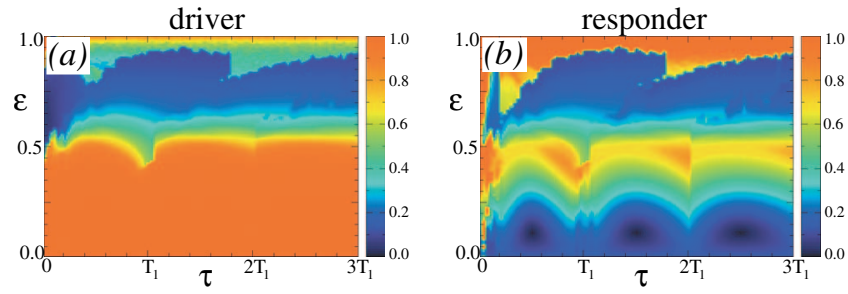

FIG. 2. (Color) Impact of the mixed NDF stimulation (2) on ensembles (1) versus delay $\tau$ and mixing parameter $\varepsilon$. The values of the time-averaged order parameters (a) $\langle R\rangle$ and (b) $\langle Q\rangle$ of the first and the second ensembles (1), respectively, are encoded by color ranging from blue (small values of the order parameters, characteristic for desynchronization) to red (large values of the order parameters, characteristic for synchronization). The stimulus amplification $K=400$, and the other parameters as in Fig. 1 .

$=300$ results in a desynchronization of the driven and stimulated ensemble 2 whose mean field becomes small. Note, without mixing, i.e., for $\varepsilon=0$ [Fig. 1(a)], the drive population is not affected by stimulation and remains strongly synchronized. Intuitively, we would expect that since the mean field of the stimulated response ensemble $W$ becomes small, it does not significantly influence the dynamics of the driver through the backward coupling $C_{21} W$, and, thus, the oscillators of the first population remain synchronized because of the strong internal coupling among them. However, the impact of the stimulation with mixed NDF, i.e., in the second case with $\varepsilon=0.73$ [Fig. 1(b)], is surprisingly different: Both ensembles are effectively desynchronized by the stimulation, indicating an effect of indirect control by the mixed NDF. Furthermore, simultaneously with the backward coupling $C_{21} W$, also the amplitude of the total external force $F=\mid S$ $+C_{12} Z$ applied to the second, driven and stimulated ensemble becomes small [Fig. 1, blue curves]. This naturally realizes the demand-controlled mode of operation of the mixed NDF stimulation, where the forcing is applied only if undesirable synchronization is present. Therefore, both populations become effectively decoupled from each other and receive a minimal amount of external force. We also note that the stimulation does not disturb the dynamics of the individual oscillators as illustrated in the subplots in Fig. 1(b), where the trajectories of two selected oscillators from both ensembles (1) in the stimulated regime are shown. The elements of the ensembles oscillate with frequencies and amplitudes close to their natural ones. The stimulation thus restores the natural dynamics of the oscillators as if they were not coupled and not forced. Below we explain in more detail the phenomena of indirect control of synchronization and decoupling of interacting ensembles by stimulation with the mixed NDF (2).

The discussed effects of the stimulation with the mixed NDF (2) are robust phenomena and are preserved for a wide range of system and stimulation parameters. This is illustrated in Fig. 2, where the time-averaged order parameters (see Refs. [2,4]) $\langle R\rangle=\left\langle\left|N_{1}^{-1} \sum_{j=1}^{N_{1}} z_{j} /\right| z_{j}||\right\rangle$ [Fig. 2(a)] and $\langle Q\rangle$ $=\left\langle\left|N_{2}^{-1} \sum_{k=1}^{N_{2}} w_{k} /\right| w_{k}||\right\rangle$ [Fig. 2(b)] of the first and the second ensembles, respectively, are encoded by color versus parameters $\tau$ and $\varepsilon$. For small values of the mixing parameter $\varepsilon$ (up to approximately 0.3 ) the mixed NDF desynchronizes the driven and stimulated population $2(\langle Q\rangle$ is small), but the driving ensemble 1 remains unaffected and exhibits strongly synchronized dynamics $(\langle R\rangle$ is large), see also Fig. 1(a). For an intermediate level of mixing both ensembles are synchronized (both $\langle R\rangle$ and $\langle Q\rangle$ are large). The indirect control of synchronization is observed for larger values of $\varepsilon$ [blue regions in Fig. 2 for approximately $0.5<\varepsilon<0.9$ ], where synchronization is suppressed in both ensembles (1) (both $\langle R\rangle$ and $\langle Q\rangle$ are small), see also Fig. 1(b).

\section{PHASE DYNAMICS}

We investigate the phase dynamics of the populations (1) in more detail. Substituting $z_{j}=r_{j} e^{i \psi_{j}}$ and $w_{k}=q_{k} e^{i \theta_{k}}$ into Eq. (1) and neglecting the amplitude dynamics, we obtain the following system of interacting phase ensembles:

$$
\begin{gathered}
\dot{\psi}_{j}=\omega_{j}+C_{1} R \sin \left(\Psi-\psi_{j}\right)+C_{21} Q \sin \left(\Theta-\psi_{j}\right), \\
\dot{\theta}_{k}=\xi_{k}+C_{2} Q \sin \left(\Theta-\theta_{k}\right)+C_{12} R \sin \left(\Psi-\theta_{k}\right)+S_{p h},
\end{gathered}
$$

where $\psi_{j}$ and $\theta_{k}$ are the phases of the individual oscillators. The mean fields of ensembles (3) are $Z=R e^{i \Psi}=N_{1}^{-1} \sum_{j=1}^{N_{1}} e^{i \psi_{j}}$ and $W=Q e^{i \Theta}=N_{2}^{-1} \sum_{k=1}^{N_{2}} e^{i \theta_{k}}$, where $R$ and $Q$ are the order parameters, and $\Psi$ and $\Theta$ are the mean phases $[2,4]$. The term $S_{p h}=|S| \sin \left(\Phi-\theta_{k}\right)$ is the corresponding phase representation of the stimulation signal $S$ from Eq. (2), where $|S|$ and $\Phi$ are the amplitude and the phase of the stimulation signal $S$, respectively. As before, we consider $C_{2}=0$ (the observed phenomena are qualitatively preserved also for a weak coupling $C_{2}>0$ ) and large $C_{1}, C_{12}$, and $C_{21}$. Then the dynamics of the mean fields $Z$ and $W$ can be modeled by a system of two coupled limit-cycle oscillators (see also Refs. [43,45,52])

$$
\begin{gathered}
\dot{Z}=i \Omega_{0} Z+\frac{C_{1}}{2}\left(1-|Z|^{2}\right) Z+\frac{C_{21}}{2}\left(W-W^{*} Z^{2}\right), \\
\dot{W}=i \Xi_{0} W+\frac{C_{12}}{2}\left[Z-Z^{*} W^{2}\right]+S / 2,
\end{gathered}
$$

where $\Omega_{0}$ and $\Xi_{0}$ are the mean frequencies of the ensembles (3), and the stimulation signal $S(t)$ models the signal $S_{p h}(t)$ from Eq. (3) and has the form (2). In the stimulation-free regime $(S=0)$, the limit cycles $Z(t)=R(t) e^{i \Psi(t)}$ and $W(t)$ $=Q(t) e^{i \Theta(t)}$ of Eq. (4) are phase locked with the amplitudes $R(t)=Q(t) \equiv 1$ indicating a strong synchronization of ensembles (3).

We show that the model system (4) gives a good approximation of the mean fields of the stimulated ensembles (3). To simplify our consideration, we assume $\Xi_{0}=\Omega_{0}$. The detailed analysis of Eq. (4) is somewhat involved, but for the delays $\tau=T / 2,3 T / 2,5 T / 2, \ldots$, where $T=2 \pi / \Omega_{0}$ is the mean period of ensembles (3), we can analytically derive solutions $Z(t)$ and $W(t)$ of Eq. (4). We find that for a range of the mixing parameter $\varepsilon$ the limit-cycle oscillators (4) are phase locked at the frequency $\Omega_{0}$ with a phase shift $\alpha=\Theta-\Psi$ which depends on the values of $\varepsilon$. Then the constant amplitudes $R$ and $Q$ of the limit cycles (4), which model the dynamics of the order parameters of ensembles (3), can be found from 

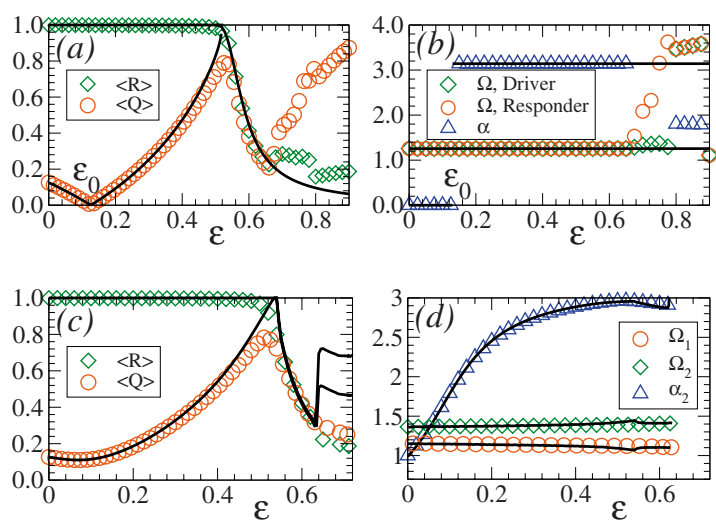

FIG. 3. (Color online) Desynchronization by mixed NDF: numerical simulation versus theoretical predictions. Symbols depict values of the time-averaged order parameters $\langle R\rangle$ and $\langle Q\rangle$, frequencies of the mean fields $\Omega$, and the phase shift $\alpha$ between phaselocked mean fields $Z$ and $W$ numerically calculated for ensembles (3) versus the level of mixing $\varepsilon$ in the stimulation signal $S$, as indicated in the legends. The black solid curves are the corresponding theoretical approximations from Eqs. (4)-(6). In (d) two different frequencies $\Omega_{1}$ and $\Omega_{2}$ of synchronized mean fields $Z$ and $W$ are shown indicating a coexistence of two different stable regimes. Number of oscillators in Eq. (3) $N_{1}=N_{2}=200$, and the natural frequencies $\omega_{j}$ and $\xi_{k}$ are Gaussian distributed around the mean frequency $\Omega_{0}=2 \pi / T \approx 1.2566(T=5)$ with deviation $\sigma=0.05$. The other parameters $C_{2}=0, C_{1}=C_{12}=C_{21}=1 ; K=500 ; \tau=T / 2$ in (a) and (b), and $\tau=4 T$ in (c) and (d).

$$
\begin{gathered}
R=1, \quad-K(Q(1-\varepsilon) \pm \varepsilon)^{3} \pm C_{12}\left(1-Q^{2}\right)=0, \\
R=\tilde{C} Q, \quad Q^{2}=\frac{C_{12} \widetilde{C}}{K[\varepsilon(1+\widetilde{C})-1]^{3}+C_{12} \tilde{C}},
\end{gathered}
$$

were $\widetilde{C}=C_{21} / C_{1}$, and Eq. (6) holds for $\varepsilon \geq 1 /(1+\widetilde{C})$. The signs "+" and " - " in Eq. (5) correspond to the phase shift $\alpha=0$ and $\alpha=\pi$, respectively. Equation (5) has a solution $Q$ $\in[0,1]$ for $\varepsilon \in\left[0, \varepsilon_{0}\right)$ if $\alpha=0$ and for $\varepsilon \in\left(\varepsilon_{0}, \varepsilon_{1}\right)$ if $\alpha=\pi$, where $\varepsilon_{0}^{3}=C_{12} / K$, and $\varepsilon_{1}$ can be found from the equation $2 C_{12}\left[8 \varepsilon^{3}-18 \varepsilon^{2}+9 \varepsilon+\left(4 \varepsilon^{2}-6 \varepsilon+3\right)^{3 / 2}\right] /\left[27(2 \varepsilon-1)^{2}(\varepsilon-1)^{2}\right]$ $=K$.

Theoretical approximations obtained from Eqs. (4)-(6) agree well with the results of numerical simulations of the stimulated ensembles (3), as illustrated in Fig. 3. Indeed, in accordance with the theoretical predictions, the mean fields of the phase ensembles (3) are phase locked at the frequency $\Omega=\Omega_{0}$ [Fig. 3(b), red circles and green diamonds] for a range $\varepsilon<\varepsilon_{s}$ [ $\varepsilon_{s} \approx 0.67$ in Figs. 3(a) and 3(b), where $\tau=T / 2$ ]. The phase locking is in-phase (phase shift $\alpha=0$ ) for $\varepsilon<\varepsilon_{0}$ and anti-phase (phase shift $\alpha=\pi$ ) for $\varepsilon>\varepsilon_{0}$ [Fig. 3(b), blue triangles]. The order parameter $Q$ of the driven and stimulated population is small for $\varepsilon<\varepsilon_{0}$ and reaches its minimum $Q=0$ at a singular point $\varepsilon=\varepsilon_{0}$ [Fig. 3(a), red circles]. At this point a transition from in-phase to antiphase synchronization of the mean fields takes place.

For a range $\varepsilon<\varepsilon_{m}\left[\varepsilon_{m} \approx 0.5\right.$ in Fig. 3(a)] the drive population is not affected by the stimulation, and its order parameter $R$ is close to 1 [Fig. 3(a), green diamonds]. As $\varepsilon$ in-

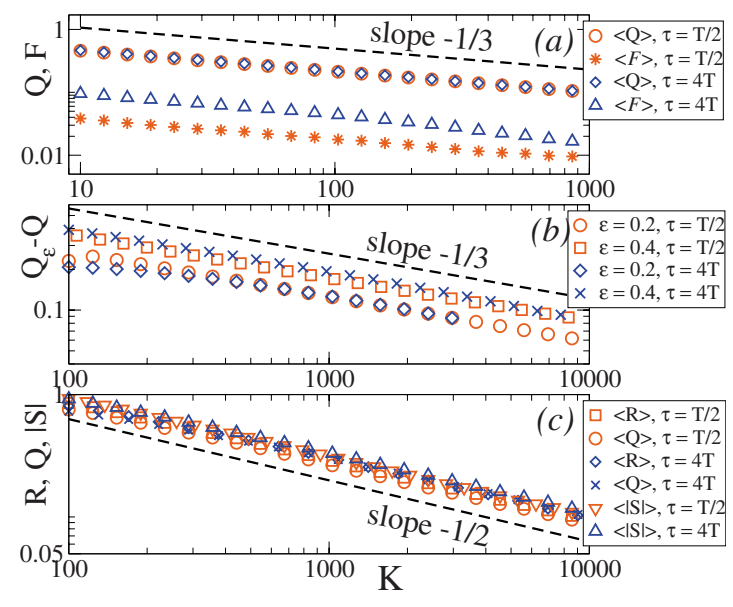

FIG. 4. (Color online) Asymptotic scaling of the time-averaged order parameters $\langle R\rangle$ and $\langle Q\rangle$, external force $\langle F\rangle$, and the amplitude of the stimulation signal $\langle|S|\rangle$ of the phase oscillators (3) versus increasing stimulus amplification $K$. Symbols depict the above quantities numerically calculated for ensembles (3), as indicated in the legends. The plots are in log-log scale, where the slopes of the black dashed lines are $-1 / 3$ in (a) and (b) and $-1 / 2$ in (c), which indicate the corresponding theoretical predictions obtained from Eqs. (4)-(6). The mixing parameter $\varepsilon=0$ in (a) and $\varepsilon=0.6$ in (c). Other parameters as in Fig. 3.

creases, the order parameter $Q$ of the driven and stimulated population 2 reaches its maximal value and then both order parameters $Q$ and $R$ start to decay [Fig. 3(a)]. This indicates an onset of desynchronization in both ensembles, see also Figs. 1 and 2. Note, if the mean fields $Z$ and $W$ of ensembles (3) are synchronized, their dynamics is well approximated by the analytically derived solutions of the model system (4) [Figs. 3(a) and 3(b), black curves].

The model system (4) also gives a good approximation for other values of the delay, which is illustrated in Figs. 3(c) and 3(d) for a large delay $\tau=4 T$ representing the case $\tau$ $=T, 2 T, 3 T, \ldots$ We find that the synchronized dynamics of the mean fields of ensembles (3) can exhibit multistability. Depending on the initial conditions both mean fields can synchronize and oscillate either at the frequency $\Omega_{1}$ or $\Omega_{2}$ [Fig. $3(\mathrm{~d})$, red circles and green diamonds]. These frequencies symmetrically relate to each other with respect to the mean natural frequency $\Omega_{0}$, i.e., $\Omega_{0}-\Omega_{1}=\Omega_{2}-\Omega_{0}$. For example, $\Omega_{1} \approx 1.1498$ and $\Omega_{2} \approx 1.3634$ for $\varepsilon=0$ in Fig. 3(d), where $\Omega_{0} \approx 1.2566$. The corresponding phase shifts $\alpha_{1}$ and $\alpha_{2}$ [Fig. 3 (d), blue triangles] are also symmetrically related with respect to $\alpha=\pi$, i.e., $\alpha_{1}=2 \pi-\alpha_{2}$.

The asymptotic dynamics of the order parameters $R$ and $Q$ of ensembles (3) as well as the amplitude of the external forcing $F=\left|S+C_{12} Z\right|$ received by the driven and stimulated population 2 is illustrated in Figs. 4(a)-4(c) versus the stimulus amplification $K$. Without mixing, i.e., for $\varepsilon=0$ and for the case $\tau=n T / 2, n=1,3,5, \ldots$, the mean fields of ensembles (3) are in-phase locked $(\alpha=\Theta-\Psi=0)$ and the stimulation signal $S(t)=|S(t)| e^{i \Phi(t)}$ is in anti-phase to the mean field of the drive population 1, i.e., $\Phi-\Psi=\pi$. From Eq. (5) we can estimate the largest term in the power-series expansion of the order parameter $Q$ of the stimulated ensemble, which gives the 
decay rate $Q \sim K^{-1 / 3}$ as $K \rightarrow \infty$. This agrees with the numerical simulations of ensembles (3) [Fig. 4(a), red circles]. Moreover, from Eq. (5) we find that the total external force $F$ applied to the driven and stimulated population also decays to zero with the rate $F \sim K^{-\gamma}$ as $K \rightarrow \infty$. This is also confirmed by numerical simulations [Fig. 4(a), red asterisks]. The evaluation of the exponent $\gamma$ however requires an estimation of the high-order terms of the power-series expansion of $Q$. Because of the approximative character of system (4), Eq. (5) gives $\gamma=2 / 3$, which differs from the value obtained in numerical simulations of ensembles (3), where $\gamma=1 / 3$ [Fig. 4(a), red asterisks]. For the case $\tau=T n, n=1,2, \ldots$, the order parameter $Q$ and the external force $F$ exhibit the same decay rates [Fig. 4(a), blue diamonds and triangles]. Therefore, with increasing $K$ the stimulated population becomes effectively desynchronized, where its order parameter decreases with the rate $K^{-1 / 3}$. Both ensembles become effectively decoupled from each other, where the amount of the residual external force and coupling between the ensembles, i.e., the amplitudes of the signals $S(t)+C_{12} Z(t)$ and $C_{21} W(t)$ decay with increasing $K$ at the same rate $K^{-1 / 3}$.

For another branch of the solution of Eq. (5), which is defined for $\varepsilon \in\left(\varepsilon_{0}, \varepsilon_{1}\right)$, the dynamics of the order parameter $Q$ is different. As follows from Eq. (5), $Q$ approaches a finite value $Q_{\varepsilon}=\varepsilon /(1-\varepsilon)$ from below as $K \rightarrow \infty$. From Eq. (5) it also follows that the difference $Q_{\varepsilon}-Q$ decays to zero with the rate $K^{-1 / 3}$ as $K \rightarrow \infty$. This is in accordance with the numerical simulations of ensembles (3) illustrated in Fig. 4(b) for $\varepsilon=0.2$ and $\varepsilon=0.4$. The total external force $F$ is bounded from above as well. For $\tau=n T / 2, n=1,3,5, \ldots$ and large $K$ $>0$, we find $F \leq C_{12} \varepsilon^{2} /(1-\varepsilon)^{2}$. We note that the mean fields of the interacting populations are anti-phase related to each other for these values of $\varepsilon$ [Fig. 3(b), blue triangles].

Finally, for a range of the mixing parameter $\varepsilon$, where both ensembles (3) are affected by the stimulation, and both order parameters $R$ and $Q$ are suppressed [Figs. 3(a) and 3(c)], the dynamics of the order parameters is approximated by Eq. (6). One can see that in this case both $R$ and $Q$ decay to zero with the rate $K^{-1 / 2}$ as $K$ increases. The amplitude of the stimulation signal $|S|$ also tends to zero with the same decay rate, which follows from the form of the stimulation signal (2) and Eq. (6). Numerical simulations of ensembles (3) confirm the theoretical predictions [Fig. 4(c)]. The above decay rate holds for both considered cases of the delay values, $\tau$ $=n T / 2, n=1,2, \ldots$, as illustrated in Fig. 4(c) for $\tau=T / 2$ [red symbols] and $\tau=4 T$ [blue symbols].

\section{ENSEMBLES OF VAN DER POL OSCILLATORS}

To demonstrate the generality of the results on synchronization control and asymptotic dynamics of the order parameters obtained for the limit-cycle (1) and phase ensembles (3), we apply the mixed NDF to a system of two interacting ensembles of relaxation van der Pol oscillators [53-55]

$$
\left\{\begin{array}{l}
\dot{x}_{j}=y_{j}, \\
\dot{y}_{j}=\mu_{j}\left(1-x_{j}^{2}\right) y_{j}-x_{j}+C_{1} Y+C_{21} V,
\end{array}\right.
$$

$$
\left\{\begin{array}{l}
\dot{u}_{k}=v_{k}, \\
\dot{v}_{k}=\eta_{k}\left(1-u_{k}^{2}\right) v_{k}-u_{k}+C_{2} V+C_{12} Y+S_{i m} .
\end{array}\right.
$$

As for the limit-cycle oscillators (1), the van der Pol oscillators are globally coupled via the mean fields $Y=N_{1}^{-1} \Sigma_{j=1}^{N_{1}} y_{j}$ and $V=N_{2}^{-1} \sum_{k=1}^{N_{2}} v_{k}$ within ensembles as well as between ensembles. The second population (variables $\left.\left(u_{k}, v_{k}\right)\right)$ is stimulated with the signal $S_{i m}(t)$ constructed according to the algorithm of the mixed nonlinear delayed feedback. For this, we consider two complex-valued mean fields $Z(t)=X(t)$ $+i Y(t)$ and $W(t)=U(t)+i V(t)$, where $X$ and $Y(U$ and $V)$ are the ensemble averages of the variables $x_{j}$ and $y_{j}\left(u_{k}\right.$ and $\left.u_{k}\right)$, respectively. Then the complex-valued stimulation signal $S(t)$ is calculated according to Eq. (2), and the stimulation signal $S_{i m}(t)$ in Eq. (7) is the imaginary part of $S(t)$, i.e., $S_{i m}(t)=\operatorname{Im}[S(t)]$. To illustrate the stimulation impact, we calculate the amplitudes of the above complex-valued mean fields $|Z|$ and $|W|$ as well as the total force administered to the second population $F=\left|S+C_{12} Z\right|$. Moreover, we also evaluate the synchronization order parameters $R$ and $Q$ of the first, drive population and the second, driven and stimulated population, respectively, which are the amplitudes of the normalized mean fields of the ensembles, see Sec. II.

Collective dynamics of oscillators (7) with mixed NDF very much resembles the dynamics of the stimulated limitcycle (1) and phase (3) oscillators. Indeed, for the limit-cycle and phase oscillators, if the mixing $\varepsilon$ in the stimulation signal is small, i.e., the driven ensemble is practically stimulated with its own mean field, the drive ensemble is not affected by the stimulation, whereas the driven and stimulated second ensemble is effectively decoupled from the driver and desynchronized [Figs. 1(a), 2, 3(a), and 3(c)]. The same holds for the van der Pol oscillators (7), which is reflected by the amplitudes of the ensembles' mean fields [Fig. 5(a), green diamonds and red circles]. Moreover, the extent of synchronization in the drive and response ensembles (7) qualitatively follows that for the phase oscillators (3) as the mixing parameter $\varepsilon$ varies [compare Figs. 5(a), 3(a), and $3(\mathrm{c})]$. In particular, both interacting ensembles get desynchronized for large $\varepsilon$. The asymptotic decay rates of the order parameters derived for the phase ensembles (3) with the use of the model system (4) [Figs. 4(a)-4(c)] also hold for the van der Pol oscillators. In particular, for $\varepsilon=0$ the order parameter $Q$ of the second, driven and stimulated ensemble decays as $K^{-1 / 3}$ if $K$ increases [Fig. 5(b), blue squares]. The order parameters $R$ and $Q$ of both interacting populations (7) obey the scaling $R, Q \sim K^{-1 / 2}$ as $K$ increases for large $\varepsilon$ [Fig. $5(\mathrm{~b})$, green diamonds and violet triangles]. Simultaneously, the amplitude of the external force $F$ administered to the second population is small [Fig. 5(a), blue triangles]. In the latter case of large $\varepsilon$, for instance, it also decays as $K^{-1 / 2}$, which directly follows from the form of the stimulation signal $S(t)$ (2) and the above scaling of the order parameters $R$ and $Q$. We also note that the delay $\tau=3$ considered in Fig. 5 is not an integer multiple of the half of the natural period $T / 2$ used for the analytical derivations in Sec. III $(T \approx 6.67$ in Fig. $5)$. Therefore, the presented desynchronizing impact of the mixed NDF is robust with respect to parameter variations, e.g., with respect to delay [see Fig. 2], level of mixing of the 

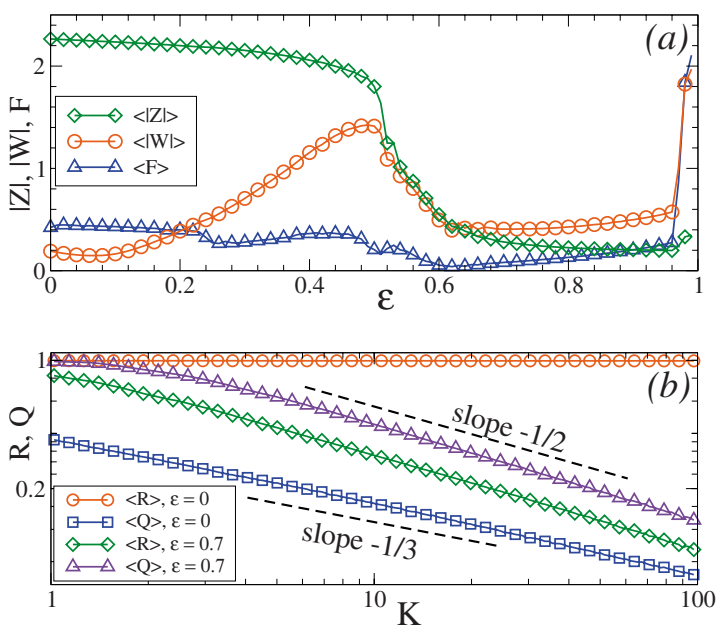

FIG. 5. (Color online) Desynchronization by mixed NDF of the two ensembles of interacting van der Pol oscillators (7). Symbols depict values of the time-averaged amplitudes of the complexvalued mean fields $\langle|Z(t)|\rangle$ and $\langle|W(t)|\rangle$ and the corresponding synchronization order parameters $\langle R\rangle$ and $\langle Q\rangle$ of the first (variables $\left(x_{j}, y_{j}\right)$ and the second (variables $\left(u_{k}, v_{k}\right)$ ensemble, respectively, as well as the time-averaged total force $\langle F\rangle$ administered to the second ensemble, as indicated in the legends. The plot (b) is in log-log scale, where the slopes of the dashed lines are $-1 / 3$ and $-1 / 2$. Number of oscillators in Eq. (7) $N_{1}=N_{2}=200$, and the parameters $\mu_{j}$ and $\eta_{j}$ are Gaussian distributed around the mean 0.7 with standard deviation $\sigma=0.1$. The coupling parameters $C_{2}=0.01$ and $C_{1}$ $=C_{12}=C_{21}=0.2$. Delay $\tau=3$, the stimulus amplification $K=50$ in (a), and the mixing parameter $\varepsilon=0,0.7$ in (b), see the legend.

mean fields in the stimulation signal, and the stimulus amplification. Accordingly, the method shows great applicability and works for ensembles of different limit cycle and phase oscillators.

\section{DISCUSSION}

In conclusion, we have presented a method for an effective decoupling and desynchronization of two interacting drive-response populations of oscillators, where the driven ensemble is stimulated with the mixed nonlinear delayed feedback. A natural desynchronized dynamics is recovered either in the stimulated ensemble or, which is most surprising, in both of them depending on the level of mixing of the measured macroscopic dynamics (mean fields) of the populations in the NDF signal. We show that the extent of synchronization in the ensembles as given by the values of the order parameters decays with increasing stimulus amplification such that the amount of the residual coupling between ensembles and the total external force administered to the stimulated ensemble also decay with the same rate. The suggested method might be of a particular importance for application, where two (or more) interacting ensembles are needed to be decoupled from each other to restore desynchronized dynamics in a demand-controlled way, and where only the driven ensemble is available or preferable for stimulation, and a measured signal is a mixture of the macroscopic dynamics of all interacting ensembles.

Besides the effective decoupling and desynchronization of the interacting ensembles, the mixed NDF can also force the synchronized ensembles to change their phase relation from in-phase to antiphase. This might be important for the generation of different patterns of neuronal dynamics, e.g., within motor circuits of the brain. For instance, this might enable to switch between different dynamical modes of pattern generators [56].

We suggest our method for the desynchronization of pathologically interacting and synchronized neuronal populations characteristic, e.g., for PD. In many experimental and clinical studies on PD it has been found that the entire cortico-basal ganglia-thalamo-cortical motor loop is affected by an abnormal neuronal synchronization [11,12,14,31]. To counteract the pathological neuronal activity, different targets for HF DBS have been empirically identified for effective open-loop single-site stimulation within the motor functional circuit $[13,32,38]$. Stimulation of the neuronal clusters within basal ganglia such as subthalamic nucleus (STN) or globus pallidus (GP) significantly reduces parkinsonian symptoms and contributes to a normalization of cortical neuronal activity $[13,32,38,57,58]$.

A number of model-based stimulation methods have been designed to effectively counteract synchronization in intrinsically synchronized oscillatory neuronal populations [40-47]. For the drive-response coupling scheme considered in the present paper previously designed linear and nonlinear feedback stimulation techniques without mixing can desynchronize the drive population only if it is directly stimulated $[59,60]$. In contrast, the considered mixed NDF can desynchronize either exclusively the stimulated ensemble, or, if the macroscopic activities of both interacting populations are appropriately mixed for the sake of stimulation, it can also desynchronize the drive ensemble, which is not stimulated at all. In the case where only different linear combinations of the mean fields of the drive and response ensembles can be measured, the desirable mixing of the signals can always be obtained by an appropriate rescaling and selection of the mixing parameter.

The mixed NDF stimulation can be a first step toward a multi-target control approach, which uses multisite recording (in different target populations) and single-site stimulation (in only one target population) to reduce the rate of side effects. In this way, appropriately chosen mixing parameters enable to switch pathological synchronization processes in different parts of the brain on and off in a well-defined, selective manner. Applying our mixed NDF approach to the parkinsonian state, the motor cortex areas, that are driven by strongly synchronized neuronal clusters from the basal ganglia $[10,11]$, could be decoupled from the latter by stimulation with the mixed NDF via an epicortical electrode without depth electrode implantation. For the calculations of the mixed stimulation signal one could use epicortical recordings (from primary motor areas and/or premotor areas) on the one 
hand and electromyographic or accelerometer signals appropriately representing the basal ganglia activity [23,24]. Such a considerably less invasive stimulation could then normalize the neuronal activity within the basal ganglia indirectly-as described above.

\section{ACKNOWLEDGMENTS}

We gratefully acknowledge discussions with Volker Hadamschek.
[1] A. T. Winfree, The Geometry of Biological Time (SpringerVerlag, Berlin, 1980).

[2] Y. Kuramoto, Chemical Oscillations, Waves, and Turbulence (Springer, Berlin, Heidelberg, New York, 1984).

[3] M. Steriade, E. G. Jones, and R. R. Llinas, Thalamic Oscillations and Signaling (Wiley, New York, 1990).

[4] P. A. Tass, Phase Resetting in Medicine and Biology: Stochastic Modelling and Data Analysis (Springer, Berlin, 1999).

[5] A. Pikovsky, M. Rosenblum, and J. Kurths, Synchronization, A Universal Concept in Nonlinear Sciences (Cambridge University Press, Cambridge, England, 2001).

[6] E. Mosekilde, Y. Maistrenko, and D. Postnov, Chaotic Synchronization: Applications to Living Systems (World Scientific, Singapore, 2002).

[7] S. H. Strogatz, Sync: The Emerging Science of Spontaneous Order (Hyperion Books, New York, 2003).

[8] A. Balanov et al., Synchronization:From Simple to Complex (Springer, New York, 2009).

[9] W. Singer and C. M. Gray, Annu. Rev. Neurosci. 18, 555 (1995).

[10] H. Bergman and G. Deuschl, Mov Disord. 17, S28 (2002).

[11] P. Brown, Mov Disord. 18, 357 (2003).

[12] A. Schnitzler and J. Gross, Nat. Rev. Neurosci. 6, 285 (2005).

[13] T. Wichmann and M. R. DeLong, Neuron 52, 197 (2006).

[14] P. Gatev, O. Darbin, and T. Wichmann, Mov Disord. 21, 1566 (2006).

[15] F. Steigerwald et al., J. Neurophysiol. 100, 2515 (2008).

[16] J. M. Hurtado et al., Proc. Natl. Acad. Sci. U.S.A. 96, 1674 (1999).

[17] R. Levy et al., J. Neurosci. 20, 7766 (2000).

[18] A. Nini et al., J. Neurophysiol. 74, 1800 (1995).

[19] H. Bergman et al., Trends Neurosci. 21, 32 (1998).

[20] C. L. Wilson, M. Puntis, and M. G. Lacey, Neuroscience 123, 187 (2004).

[21] F. Amtage et al., Neurosci. Lett. 442, 195 (2008).

[22] C. Reck et al., Eur. J. Neurosci. 29, 599 (2009).

[23] D. A. Smirnov et al., EPL 83, 20003 (2008).

[24] P. Tass et al., J. Neural Eng. 7, 016009 (2010).

[25] A. A. Kuhn et al., Eur. J. Neurosci. 23, 1956 (2006).

[26] P. J. Magill, J. P. Bolam, and M. D. Bevan, Neuroscience 106, 313 (2001).

[27] N. Fogelson et al., Cereb. Cortex 16, 64 (2006).

[28] P. Gatev and T. Wichmann, Cereb. Cortex 19, 1330 (2009).

[29] P. Tass, M. G. Rosenblum, J. Weule, J. Kurths, A. Pikovsky, J.
Volkmann, A. Schnitzler, and H. J. Freund, Phys. Rev. Lett. 81, 3291 (1998).

[30] L. Timmermann et al., Brain 126, 199 (2003).

[31] M. R. DeLong and T. Wichmann, Arch. Neurol. 64, 20 (2007).

[32] A. L. Benabid et al., Lancet 337, 403 (1991).

[33] T. Hashimoto et al., J. Neurosci. 23, 1916 (2003).

[34] M. Filali et al., Exp. Brain Res. 156, 274 (2004).

[35] C. C. McIntyre et al., Clin. Neurophysiol. 115, 1239 (2004).

[36] P. R. Schuurman et al., N. Engl. J. Med. 342, 461 (2000).

[37] R. Kumar et al., Neurology 61, 1601 (2003).

[38] J. Volkmann, J. Clin. Neurophysiol. 21, 6 (2004).

[39] M. C. Rodriguez-Oroz et al., Brain 128, 2240 (2005).

[40] P. A. Tass, Biol. Cybern. 89, 81 (2003).

[41] P. A. Tass, Prog. Theor. Phys. 150, 281 (2003).

[42] M. G. Rosenblum and A. S. Pikovsky, Phys. Rev. Lett. 92, 114102 (2004).

[43] O. V. Popovych, C. Hauptmann, and P. A. Tass, Phys. Rev. Lett. 94, 164102 (2005).

[44] C. Hauptmann, O. Popovych, and P. A. Tass, Neurocomputing 65-66, 759 (2005).

[45] O. V. Popovych, C. Hauptmann, and P. A. Tass, Biol. Cybern. 95, 69 (2006).

[46] K. Pyragas, O. V. Popovych, and P. A. Tass, EPL 80, 40002 (2007).

[47] I. Z. Kiss et al., Science 316, 1886 (2007).

[48] A. B. Neiman, D. F. Russell, T. A. Yakusheva, A. DiLullo, and P. A. Tass, Phys. Rev. E 76, 021908 (2007).

[49] P. A. Tass and M. Majtanik, Biol. Cybern. 94, 58 (2006).

[50] M. Hämäläinen et al., Rev. Mod. Phys. 65, 413 (1993).

[51] Y. A. Kuznetsov, Elements of Applied Bifurcation Theory (Springer, New York, 1998).

[52] C. J. Perez and F. Ritort, J. Phys. A 30, 8095 (1997).

[53] B. van der Pol, Radio Rev. 1, 704 (1920).

[54] B. van der Pol, Philos. Mag. J. Sci. Ser. 7 3, 65 (1927).

[55] B. van der Pol and J. van der Mark, Philos. Mag. 6, 763 (1928).

[56] C. Hauptmann, O. Omelchenko, O. V. Popovych, Y. Maistrenko, and P. A. Tass, Phys. Rev. E 76, 066209 (2007).

[57] S. T. Grafton et al., Neurology 66, 1192 (2006).

[58] P. Silberstein et al., Brain 128, 1277 (2005).

[59] C. Hauptmann, O. Popovych, and P. A. Tass, Biol. Cybern. 93, 463 (2005).

[60] O. V. Popovych, C. Hauptmann, and P. A. Tass, Int. J. Bifurcation Chaos Appl. Sci. Eng. 16, 1977 (2006). 